\title{
Active Flux Schemes for Diffusion
}

\author{
Hiroaki Nishikawa * \\ National Institute of Aerospace, Hampton, VA 23666 \\ Philip L. Roe ${ }^{\dagger}$ \\ Department of Aerospace Engineering, University of Michigan, Ann Arbor, MI 48109 \\ Timothy A. Eymann $\ddagger$ \\ DoD HPCMP/CREATE Kestrel Team, Eglin AFB, FL 32542
}

\begin{abstract}
In this paper, we construct active flux schemes for diffusion. Active flux schemes are efficient third-order finite-volume-type schemes developed thus far for hyperbolic systems. This paper extends the active flux schemes to diffusion problems by the first-order hyperbolic system method, in which a numerical scheme is constructed based on a first-order hyperbolic system that is equivalent to the diffusion equation in the steady state. Active flux schemes are first developed for a generic hyperbolic system with source term, and then immediately applied to the hyperbolized diffusion system to generate a steady-state solver. Time-accurate schemes are constructed by implicit time integration where the steady-state solver is employed to solve the implicit residual equations. Numerical results show that third-order accuracy is obtained in both the solution and the gradient on irregular grids.
\end{abstract}

\section{Introduction}

In this paper, we construct active flux schemes for diffusion. Active flux schemes have been developed for hyperbolic systems of conservation laws in Refs.1,2, as a viable alternative to high-order methods. Active flux schemes are finite-volume-based compact high-order schemes. These schemes are substantially different from other high-order schemes and have attractive features for a practical implementation. First, active flux schemes do not rely on a typical one-dimensional flux across a control-volume face, but incorporate multi-dimensional physics into the residual. The numerical flux at a face is determined not by solving a one-dimensional Riemann problem, but calculated by the method of spherical mean, which is an exact solution to a multi-dimensional initial-value problem (it is equivalent to a solution to the characteristic equations in one dimension). Second, the memory requirement is much reduced compared with DG schemes due to sharing of degrees of freedom among elements. In addition to cell-averages, active flux schemes carry point-values at faces; the latter are shared by adjacent cells, thus resulting in 2 degrees of freedom per cell (for third-order accuracy) in one dimension, and approximately 3.5 in two and three dimensions. The active flux methodology has been developed for systems of hyperbolic conservation laws in Refs.1,2, but its extension to diffusive equations has not been well studied yet. Towards the development of practical third-order active-flux schemes for viscous flow simulations, in this paper, we focus on the construction of active-flux schemes for diffusion.

One possible approach to the construction of active flux schemes for diffusion is the recovery approach proposed in Ref.3. Specifically, a quartic polynomial is constructed over two adjacent cells, based on a quadratic polynomial defined within each cell, and a diffusive flux is directly evaluated by differentiation at the face. However, our experience shows that the resulting explicit time-stepping scheme is subject to a severe stability restriction, and thus limiting its potential use (an analysis is given Ref.4). In order to develop high-order diffusion schemes while preserving the advantages of the active flux scheme, we consider the construction of diffusion schemes based on the first-order hyperbolic system method where the diffusion equation is discretized in the form of a first-order hyperbolic system. One of the advantages of this method is that schemes developed for hyperbolic systems can be directly applied to diffusion. The method was first introduced in Ref.5, extended to

\footnotetext{
*Associate Research Fellow (hiro@nianet.org), National Institute of Aerospace, 100 Exploration Way, Hampton, VA 23666, Senior Member AIAA

${ }^{\dagger}$ Professor (philroe@umich.edu), University of Michigan, Ann Arbor, MI 48105, Fellow AIAA

${ }^{\ddagger}$ Research Engineer, Member AIAA.
} 
the advection-diffusion equation in Ref.6, to the Navier-Stokes equations in Ref.7, to time-dependent advectiondiffusion problems in Ref.8, and to high-order schemes (up to 6th-order) in Ref.9. At the same time, the method has also been employed for the development of first-, second-, and third-order edge-based finite-volume schemes as in Refs.10-12. Through the series of papers, the method has been shown to offer a number of advantages over conventional methods, not only the drastic simplification in discretization (i.e., advection scheme for diffusion), but also significant acceleration in steady convergence by explicit and implicit solvers, and the equal order of accuracy for the solution and the gradients (viscous/heat fluxes) on fully irregular grids. This paper demonstrates that the active flux scheme for diffusion can be constructed by applying the active flux scheme developed for hyperbolic systems as presented in Ref.1,2 in combination with the physical time integration by the backward difference formulas. In doing so, we have found that active flux schemes need a careful construction for hyperbolic systems with source terms, which include the hyperbolic diffusion system. This paper shows how

to construct active flux schemes for hyperbolic systems with source terms, and then how the resulting schemes can be immediately turned into diffusion schemes.

\section{Hyperbolic Diffusion System}

Consider the diffusion equation:

$$
u_{\tau}=\nu u_{x x}+s_{1},
$$

where $\nu$ is a constant diffusion coefficient and $s_{1}=s_{1}(x)$ is a source term. In this section, we focus on steady problems, and thus the variable $\tau$ is a pseudo time. Steady solutions can be obtained by solving, instead of the diffusion equation, the following first-order hyperbolic system:

$$
\mathbf{u}_{\tau}+\mathbf{f}_{x}=\mathbf{s},
$$

where

$$
\mathbf{u}=\left[\begin{array}{l}
u \\
p
\end{array}\right], \quad \mathbf{f}=\left[\begin{array}{c}
-\nu p \\
-u / T_{r}
\end{array}\right], \quad \mathbf{s}=\left[\begin{array}{l}
s_{1} \\
s_{2}
\end{array}\right],
$$

where $s_{2}=-p / T_{r}$. Note that the system is equivalent to the diffusion equation in the steady state for any nonzero value of the relaxation time, $T_{r}$. The equivalence in the steady state is the key idea as first proposed in Ref.5 for constructing diffusion schemes. Therefore, the relaxation time does not have to be determined by any physical consideration, but can be determined solely by numerical consideration, e.g., fast convergence to the steady state. A typical choice is the following:

$$
T_{r}=\frac{L_{r}^{2}}{\nu}, \quad L_{r}=\frac{1}{2 \pi},
$$

which can be derived by requiring Fourier modes to propagate to enhance the iterative convergence as discussed in Ref.6. The same choice has been derived based on a similar argument applied to a first-order finite-volume scheme as described in Ref.11.

Consider the flux Jacobian, $\mathbf{A}=\partial \mathbf{f} / \partial \mathbf{u}$,

$$
\mathbf{A}=\left[\begin{array}{cc}
0 & -\nu \\
-1 / T_{r} & 0
\end{array}\right]
$$

which has the following real eigenvalues:

$$
\lambda_{1}=-\lambda, \quad \lambda_{2}=\lambda, \quad \lambda=\sqrt{\frac{\nu}{T_{r}}}=\frac{\nu}{L_{r}},
$$

and linearly independent right and left eigenvectors,

$$
\mathbf{R}=\frac{1}{2}\left[\begin{array}{cc}
1 & -1 \\
1 / L_{r} & 1 / L_{r}
\end{array}\right], \quad \mathbf{L}=\mathbf{R}^{-1}=\left[\begin{array}{cc}
1 & L_{r} \\
-1 & L_{r}
\end{array}\right]
$$


Therefore, the system is hyperbolic, which describes a symmetric wave. Consequently, the system can be diagonalized by multiplying it by $\mathbf{L}$ from the left, which yields the set of characteristic equations:

$$
\partial_{\tau} w_{1}+\lambda_{1} \partial_{x} w_{1}=s_{1}^{w}, \quad \partial_{\tau} w_{2}+\lambda_{2} \partial_{x} w_{2}=s_{2}^{w},
$$

where

$$
\begin{gathered}
w_{1}=L_{r} p+u, \quad w_{2}=L_{r} p-u, \\
s_{1}^{w}=L_{r} s_{2}+s_{1}, \quad s_{2}^{w}=L_{r} s_{2}-s_{1} .
\end{gathered}
$$

Active flux schemes developed for hyperbolic systems can be directly applied to the hyperbolic diffusion system. In the steady state, we obtain the solution to the diffusion equation. However, the hyperbolic diffusion system has a source term; the one in the second equation of Eq.(2.3), i.e., $s_{2}$, is intrinsic to the hyperbolic diffusion system. Previous studies did not consider hyperbolic systems with source terms, and it has been found that active flux schemes require a careful construction for such systems. Also, it has been observed that improperly implemented boundary conditions can degrade the design accuracy of active flux schemes. In the next section, we discuss these issues in details for a generic hyperbolic system with source terms.

\section{Active Flux Scheme for Hyperbolic System with Source Terms}

\subsection{Hyperbolic System with Source Terms}

Consider a $2 \times 2$ hyperbolic system with source terms in $x \in(0,1)$ :

$$
\begin{aligned}
& u_{\tau}-a v_{x}=s_{u}(x), \\
& v_{\tau}-a u_{x}=s_{v}(x),
\end{aligned}
$$

where $\tau$ is a pseudo time, $a$ is a positive constant, and $s_{u}$ and $s_{v}$ are source terms. We are interested to compute the steady state solution with the Dirichlet boundary conditions:

$$
u(0)=u(1)=v(0)=v(1)=0 .
$$

Write the system in the vector form:

$$
\mathbf{u}_{\tau}+\mathbf{f}_{x}=\mathbf{s}
$$

where

$$
\mathbf{u}=\left[\begin{array}{l}
u \\
v
\end{array}\right], \quad \mathbf{f}=\left[\begin{array}{l}
-a v \\
-a u
\end{array}\right], \quad \mathbf{s}=\left[\begin{array}{l}
s_{u} \\
s_{v}
\end{array}\right] .
$$

The flux Jacobian, $\mathbf{A}=\partial \mathbf{f} / \partial \mathbf{u}$,

$$
\mathbf{A}=\left[\begin{array}{cc}
0 & -a \\
-a & 0
\end{array}\right]
$$

has the following eigenvalues:

$$
\lambda_{1}=-a, \quad \lambda_{2}=a,
$$

and the right and left eigenvectors,

$$
\mathbf{R}=\frac{1}{2}\left[\begin{array}{cc}
1 & 1 \\
1 & -1
\end{array}\right], \quad \mathbf{L}=\mathbf{R}^{-1}=\left[\begin{array}{cc}
1 & 1 \\
1 & -1
\end{array}\right] .
$$

The characteristic equations are obtained by multiplying the system by $\mathbf{L}$ from the left:

$$
\mathbf{w}_{\tau}+\Lambda \mathbf{w}_{x}=\mathbf{s}^{w},
$$


where $\mathbf{w}=\left(w_{1}, w_{2}\right)$, and

$$
\mathbf{w}=\mathbf{L u}=\left[\begin{array}{c}
u+v \\
u-v
\end{array}\right], \quad \mathbf{\Lambda}=\left[\begin{array}{cc}
-a & 0 \\
0 & a
\end{array}\right], \quad \mathbf{s}^{w}=\mathbf{L} \mathbf{s} .
$$

The characteristic system consists of two advection equations:

$$
\partial_{\tau}(u+v)-a \partial_{x}(u+v)=s_{1}^{w}, \quad \partial_{\tau}(u-v)+a \partial_{x}(u-v)=s_{2}^{w},
$$

where $s_{1}^{w}=s_{u}+s_{v}$ and $s_{2}^{w}=s_{u}-s_{v}$.

\subsection{Discretization}

To discretize the system by the active flux scheme, we begin by storing the cell-averages within each cell and the point-values at each face in a one-dimensional grid. In each cell, we construct quadratic polynomials, $u(x)$ and $v(x)$, by interpolating the face values and requiring that the cell-average of the polynomial reduces to the cell-averaged solution. Integrating the system (3.4) over a space-(pseudo-)time control volume, we obtain

$$
\left(\overline{\mathbf{u}}_{j}^{k+1}-\overline{\mathbf{u}}_{j}^{k}\right) h=-\Delta \tau\left[\mathbf{f}_{j+1 / 2}-\mathbf{f}_{j-1 / 2}\right]+\Delta \tau \int_{x_{j}-h / 2}^{x_{j}+h / 2} \mathbf{s} d x .
$$

Note that $\mathbf{f}_{j+1 / 2}$ and $\mathbf{f}_{j-1 / 2}$ are time-averaged fluxes, which can be evaluated by Simpson's rule and that the source term is independent of time. It leads to a pseudo-time marching scheme:

$$
\overline{\mathbf{u}}_{j}^{k+1}=\overline{\mathbf{u}}_{j}^{k}-\frac{\Delta \tau}{h} \mathbf{R e s}_{j}
$$

where

$$
\boldsymbol{R e s}_{j}=\mathbf{f}_{j+1 / 2}-\mathbf{f}_{j-1 / 2}-\int_{x_{j}-h / 2}^{x_{j}+h / 2} \mathbf{s} d x .
$$

At this point, the discretization of the source terms is straightforward. It is the interface flux step where a special treatment is required.

In active flux schemes, the interface flux is computed by the solution at face obtained by integrating the characteristic equations along the characteristics. For example, at face $j+1 / 2$, we obtain

$$
w_{1}\left(x_{j+1 / 2}\right)=w_{1}\left(x_{R}\right)+\frac{1}{\lambda_{1}} \int_{x_{R}}^{x_{j+1 / 2}} s_{1}^{w} d x, \quad w_{2}\left(x_{j+1 / 2}\right)=w_{2}\left(x_{L}\right)+\frac{1}{\lambda_{2}} \int_{x_{L}}^{x_{j+1 / 2}} s_{2}^{w} d x,
$$

where $x_{L}=x_{j+1 / 2}-\sigma h$ is the location on the left cell at time level $k$ found by tracing back the characteristic from the face, and $x_{R}=x_{j+1 / 2}+\sigma h$ is the location on the right cell found by tracing back the other characteristic. See Figure 1, which illustrates the case $j=1$. Note that $\sigma=K a \Delta \tau / h$ with $K \in(0,1]$. The source term integration can be carried out by Trapezoidal rule:

$$
\begin{gathered}
\int_{x_{R}}^{x_{j+1 / 2}} s_{1}^{w} d x=\frac{1}{2}\left[s_{1}^{w}\left(x_{j+1 / 2}\right)+s_{1}^{w}\left(x_{R}\right)\right]\left(x_{j+1 / 2}-x_{R}\right) \\
\int_{x_{L}}^{x_{j+1 / 2}} s_{2}^{w} d x=\frac{1}{2}\left[s_{2}^{w}\left(x_{j+1 / 2}\right)+s_{2}^{w}\left(x_{L}\right)\right]\left(x_{j+1 / 2}-x_{L}\right) .
\end{gathered}
$$

The system of characteristic equations (3.15) can be written in terms of the original variables as

$$
\begin{aligned}
& u\left(x_{j+1 / 2}\right)+v\left(x_{j+1 / 2}\right)=u\left(x_{R}\right)+v\left(x_{R}\right)+\frac{1}{\lambda_{1}} \int_{x_{R}}^{x_{j+1 / 2}} s_{1}^{w} d x \\
& u\left(x_{j+1 / 2}\right)-v\left(x_{j+1 / 2}\right)=u\left(x_{L}\right)-v\left(x_{L}\right)+\frac{1}{\lambda_{2}} \int_{x_{L}}^{x_{j+1 / 2}} s_{2}^{w} d x .
\end{aligned}
$$




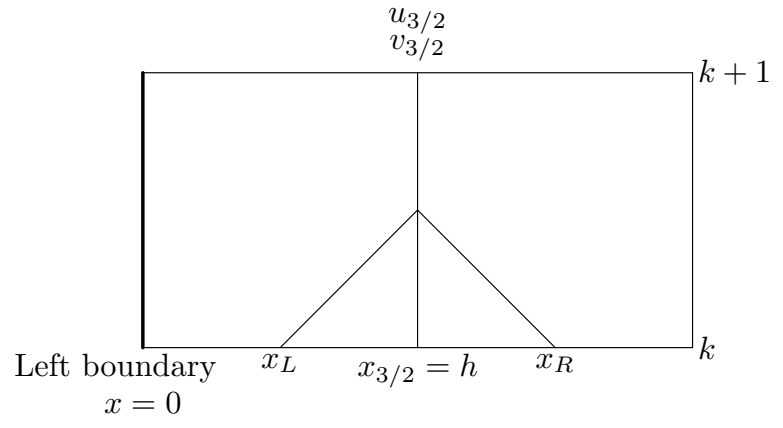

Figure 1. Finding the right face values $u_{3 / 2}$ and $v_{3 / 2}$ for the cell $j=1$, which is the left cell.

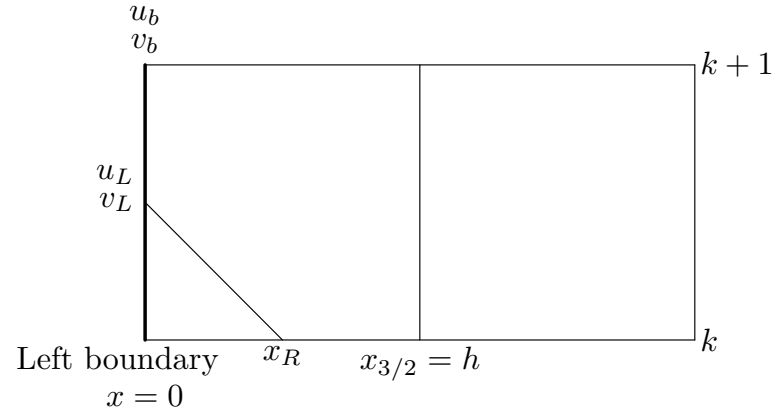

Figure 2. Finding the boundary values $u_{b}$ and $v_{b}$ for the cell $j=1$. Boundary condition is imposed on $u_{L}$ and $v_{L}$

The two equations can be solved for $u$ and $v$ at the face (add and subtract):

$$
\begin{aligned}
& u\left(x_{j+1 / 2}\right)=\frac{1}{2}\left[u\left(x_{R}\right)+u\left(x_{L}\right)+v\left(x_{R}\right)-v\left(x_{L}\right)+\frac{1}{\lambda_{1}} \int_{x_{R}}^{x_{j+1 / 2}} s_{1}^{w} d x+\frac{1}{\lambda_{2}} \int_{x_{L}}^{x_{j+1 / 2}} s_{2}^{w} d x\right], \\
& v\left(x_{j+1 / 2}\right)=\frac{1}{2}\left[v\left(x_{R}\right)+v\left(x_{L}\right)+u\left(x_{R}\right)-u\left(x_{L}\right)+\frac{1}{\lambda_{1}} \int_{x_{R}}^{x_{j+1 / 2}} s_{1}^{w} d x-\frac{1}{\lambda_{2}} \int_{x_{L}}^{x_{j+1 / 2}} s_{2}^{w} d x\right],
\end{aligned}
$$

which provide the solution at any time between $\tau$ and $\tau+\Delta \tau$ by varying $K$. For our purpose, we take $K=0.5$ to get the solution at $k+1 / 2$ and $K=1.0$ to get the solution at $k+1$ (for Simpson's rule). We remark that the integration of the source terms along the characteristics is critical to keep the third-order accuracy of the active flux scheme. The time-averaged interface flux is, therefore, given by

$$
\mathbf{f}_{j+1 / 2}=\left[\begin{array}{c}
-a \frac{v_{j+1 / 2}^{k}+4 v_{j+1 / 2}^{k+1 / 2}+v_{j+1 / 2}^{k+1}}{6} \\
-a \frac{u_{j+1 / 2}^{k}+4 u_{j+1 / 2}^{k+1 / 2}+u_{j+1 / 2}^{k+1}}{6}
\end{array}\right] .
$$

For steady computations, we may employ Trapezoidal rule instead of Simpson's rule without losing accuracy:

$$
\mathbf{f}_{j+1 / 2}=\left[\begin{array}{c}
-a \frac{v_{j+1 / 2}^{k}+v_{j+1 / 2}^{k+1}}{2} \\
-a \frac{u_{j+1 / 2}^{k}+u_{j+1 / 2}^{k+1}}{2}
\end{array}\right] .
$$

This will simplify the algorithm and reduce the cost since the half-time solution does not need to be computed. This completely defines the active flux scheme for the $2 \times 2$ hyperbolic system with source terms in the interior cells.

\subsection{Boundary Conditions}

In the cells adjacent to boundaries, the active flux scheme involves interface fluxes across boundaries. Consider the cell $j=1$ adjacent to the left boundary $(x=0)$. The steady residual is given by

$$
\boldsymbol{R e s}_{1}=\mathbf{f}_{3 / 2}-\mathbf{f}_{b}-\int_{0}^{h} \mathbf{s} d x=0
$$

where $\mathbf{f}_{b}$ is the boundary flux on the left of the cell. The flux $\mathbf{f}_{3 / 2}$ is computed as described in the previous section (see Figure 1). If the boundary flux is fixed by the boundary condition, $\mathbf{f}_{b}=0$, then we are left with

$$
\boldsymbol{R e s}_{1}=\mathbf{f}_{3 / 2}-\int_{0}^{h} \mathbf{s} d x=0 .
$$

This equation cannot be solved for the cell-averages, $\overline{\mathbf{u}}_{1}$ and $\overline{\mathbf{v}}_{1}$, because it involves only the combination $\overline{\mathbf{u}}_{1}-\overline{\mathbf{v}}_{1}$ (see Eqs.(3.20) and (3.21) with $j=1$ ). In other words, it vanishes for infinitely many solutions that differ by a 
constant. Consequently, the scheme admits infinitely many solutions. A solution to the non-uniqueness problem is to specify the boundary conditions on the outer side of the boundary face (see Figure 2$): \operatorname{set} u\left(x_{L}\right)=v\left(x_{L}\right)=0$ and get the boundary values from the characteristic equations Eqs.(3.20) and (3.21):

$$
\begin{aligned}
& u_{b}=\frac{1}{2}\left[u\left(x_{R}\right)+v\left(x_{R}\right)+\frac{1}{\lambda_{1}} \int_{x_{R}}^{x_{j+1 / 2}} s_{1}^{w} d x\right], \\
& v_{b}=\frac{1}{2}\left[v\left(x_{R}\right)+u\left(x_{R}\right)+\frac{1}{\lambda_{1}} \int_{x_{R}}^{x_{j+1 / 2}} s_{1}^{w} d x\right],
\end{aligned}
$$

where the source term integration has been ignored on the left cell that does not exist, and $R$ denotes the cell $j=1$ that is on the right side of the boundary face. In fact, this is a widely-used boundary procedure in finite-volume methods: specify the outer value and let a numerical flux determine the boundary solution. The boundary flux $\mathbf{f}_{b}$ evaluated by these values will provide coupling between $\overline{\mathbf{u}}_{1}$ and $\overline{\mathbf{v}}_{1}$ in the residual equation:

$$
\boldsymbol{R e s}_{1}=\mathbf{f}_{3 / 2}-\mathbf{f}_{b}-\int_{0}^{h} \mathbf{s} d x=0,
$$

which can now be solved to uniquely determine $\overline{\mathbf{u}}_{1}$ and $\overline{\mathbf{v}}_{1}$.

The same procedure can be applied to the cell adjacent to the right boundary. This method gives thirdorder accurate face values (including boundary values) as well as cell-averages in the steady state for any initial solution. It is simple to implement especially because we can use the same algorithm at all faces.

\section{Active Flux Scheme for Diffusion}

The hyperbolic diffusion system is a $2 \times 2$ hyperbolic system with a source term. Therefore, it can be discretized in the same way as described in the previous section. Simply following the steps illustrated by Equations (3.15)-(3.21), we obtain

$$
\begin{gathered}
u\left(x_{j+1 / 2}\right)=\frac{1}{2}\left[u\left(x_{R}\right)+u\left(x_{L}\right)+L_{r}\left(p\left(x_{R}\right)-p\left(x_{L}\right)\right)+\frac{1}{\lambda_{1}} \int_{x_{R}}^{x_{j+1 / 2}} s_{1}^{w} d x+\frac{1}{\lambda_{2}} \int_{x_{L}}^{x_{j+1 / 2}} s_{2}^{w} d x\right], \\
p\left(x_{j+1 / 2}\right)=\frac{1}{2}\left[p\left(x_{R}\right)+p\left(x_{L}\right)+\frac{1}{L_{r}}\left(u\left(x_{R}\right)-u\left(x_{L}\right)+\frac{1}{\lambda_{1}} \int_{x_{R}}^{x_{j+1 / 2}} s_{1}^{w} d x-\frac{1}{\lambda_{2}} \int_{x_{L}}^{x_{j+1 / 2}} s_{2}^{w} d x\right)\right],
\end{gathered}
$$

which are used to compute the face values, $u_{j+1 / 2}^{k+1}$ and $p_{j+1 / 2}^{k+1}$. The steady solution is computed by marching in the pseudo time:

$$
\overline{\mathbf{u}}_{j}^{k+1}=\overline{\mathbf{u}}_{j}^{k}-\frac{\Delta \tau}{h} \boldsymbol{R e s}_{j}
$$

where

$$
\begin{gathered}
\operatorname{Res}=\mathbf{f}_{j+1 / 2}-\mathbf{f}_{j-1 / 2}-\int_{x_{j}-h / 2}^{x_{j}+h / 2} \mathbf{s}^{k+1} d x, \\
\mathbf{f}_{j+1 / 2}=\left[\begin{array}{c}
-\nu p_{j+1 / 2} \\
-u_{j+1 / 2}
\end{array}\right]=\left[\begin{array}{c}
-\nu \frac{p_{j+1 / 2}^{k}+p_{j+1 / 2}^{k+1}}{2} \\
-\frac{u_{j+1 / 2}^{k}+u_{j+1 / 2}^{k+1}}{2 T_{r}}
\end{array}\right] .
\end{gathered}
$$

The source term integration in the residual can be evaluated by Simpson's rule for $s_{1}$ and exactly for $p$ that is quadratic:

$$
\int_{x_{j}-h / 2}^{x_{j}+h / 2} \mathbf{s}^{k+1} d x=\left[\begin{array}{c}
\bar{s}_{1 j} \\
-\bar{p}_{j}^{k+1} h / T_{r}
\end{array}\right],
$$


where

$$
\bar{s}_{1 j}=\frac{s_{1}\left(x_{j+1 / 2}\right)+4 s_{1}\left(x_{j}\right)+s_{1}\left(x_{j-1 / 2}\right)}{6} h .
$$

The resulting time-marching scheme is

$$
\begin{aligned}
& \bar{u}_{j}^{k+1}=\bar{u}_{j}^{k}+\frac{\Delta \tau}{h}\left(\nu p_{j+1 / 2}-\nu p_{j-1 / 2}+\bar{s}_{1 j} h\right), \\
& \bar{p}_{j}^{k+1}=\bar{p}_{j}^{k}+\frac{\Delta \tau}{h}\left(u_{j+1 / 2}-u_{j-1 / 2}-\bar{p}_{j}^{k+1} h\right) / T_{r} .
\end{aligned}
$$

The second equation may be arranged into a point-implicit form by taking the source term on the right hand side to the left hand side.

Typically, two values are specified at the boundaries, e.g., $u(0)=\alpha$ and $u(1)=\beta$ or $u_{x}(0)=\gamma$ and $u(1)=\beta$, etc., where $\alpha, \beta$, and $\gamma$ are constants. In the hyperbolic method, all conditions are of Dirichlet type because the condition on the gradient, such as $u_{x}(0)=\gamma$, can be imposed via the variable $p$ as $p(0)=\gamma$. In any case, there will always be one condition per boundary, which fixes one of the two variables. The other variable is then determined by the scheme, and may involve the solution at the previous time step. For example, on the left boundary face, we specify $u\left(x_{L}\right)=\alpha$ as a boundary condition, and set

$$
p\left(x_{L}\right)=p_{b}^{k}
$$

where $p_{b}^{k}$ is the variable $p$ at the face obtained at the previous time step.

\section{Physical Time Integration}

In the hyperbolic method, time-accurate computation is possible by implicit time integration schemes as demonstrated in Ref.8 for residual-distribution schemes. Here, we follow Ref.8 and employ the backward difference formulas (BDF) with the steady solver described in the previous section to solve the unsteady residual equations. It results in the following dual-time scheme:

$$
\begin{aligned}
& \bar{u}_{j}^{k+1}=\bar{u}_{j}^{k}+\frac{\Delta \tau}{h}\left(\nu p_{j+1 / 2}-\nu p_{j-1 / 2}-\partial_{t} \bar{u}^{k+1} h\right), \\
& \bar{p}_{j}^{k+1}=\bar{p}_{j}^{k}+\frac{\Delta \tau}{h}\left(u_{j+1 / 2}-u_{j-1 / 2}-\bar{p}_{j}^{k+1} h\right) / T_{r} .
\end{aligned}
$$

where $k$ is the inner iteration counter, and the physical time derivative is given by the BDF formula:

$$
\partial_{t} \bar{u}^{k+1}=\frac{\alpha u_{j}^{k+1}+\alpha_{n-1} \bar{u}_{j}^{n}+\alpha_{n-1} \bar{u}_{j}^{n-1}+\alpha_{n-2} \bar{u}_{j}^{n-2}}{\Delta t},
$$

for a constant physical time step $\Delta t$. The first-order accurate BDF (BDF1) is given by

$$
\alpha=1, \quad \alpha_{n}=-1, \quad \alpha_{n-1}=0, \quad \alpha_{n-2}=0,
$$

the second-order BDF (BDF2) is given by

$$
\alpha=3 / 2, \quad \alpha_{n}=-2, \quad \alpha_{n-1}=1 / 2, \quad \alpha_{n-2}=0,
$$

and the third-order BDF (BDF3) is given by

$$
\alpha=11 / 6, \quad \alpha_{n}=-3, \quad \alpha_{n-1}=3 / 2, \quad \alpha_{n-2}=-1 / 3 .
$$

The pseudo time step is defined by

$$
\Delta \tau=\mathrm{CFL} \frac{h_{m i n}}{\lambda}=\mathrm{CFL} \frac{h_{m i n}}{\sqrt{\nu / T_{r}}}
$$


where $h_{\text {min }}$ is the minimum cell volume. Once the inner iteration converges, i.e., $\bar{u}_{j}^{k+1}=\bar{u}_{j}^{k}$ and $\bar{p}_{j}^{k+1}=\bar{p}_{j}^{k}$, we obtain the solution at the next physical time level, $n+1$,

$$
\begin{aligned}
& \bar{u}_{j}^{n+1}=\bar{u}_{j}^{k+1}, \\
& \bar{p}_{j}^{n+1}=\bar{p}_{j}^{k+1} .
\end{aligned}
$$

Note that the physical time derivative term can be treated as a source term to the first equation. Therefore, the computation of the face values can be performed exactly as described in the previous section if the physical time derivative is added to the source term.

$$
s_{1} \rightarrow s_{1}-\partial_{t} u^{k+1} .
$$

It is well known since Ref.13 that the inner iteration should be performed by treating the cell-averages on the right hand side implicitly (point implicit) for stability if the explicit time-marching is employed. The source term in the second equation can also be treated point-implicitly. The sub-iteration is, therefore, performed actually in the following form:

$$
\begin{aligned}
\bar{u}_{j}^{k+1} & =\frac{1}{1+\alpha \Delta \tau / \Delta t}\left[\bar{u}_{j}^{k}+\Delta \tau\left(\frac{\nu p_{j+1 / 2}-\nu p_{j-1 / 2}}{h}-\frac{\alpha_{n-1} u_{j}^{n}+\alpha_{n-1} u_{j}^{n-1}+\alpha_{n-2} u_{j}^{n-2}}{\Delta t}\right)\right] \\
\bar{p}_{j}^{k+1} & =\frac{1}{1+\Delta \tau / T_{r}}\left[\bar{p}_{j}^{k}+\Delta \tau\left(\frac{u_{j+1 / 2}-u_{j-1 / 2}}{h T_{r}}\right)\right] .
\end{aligned}
$$

Experimentally, we found that time accuracy is obtained by two orders of magnitude reduction in the unsteady residuals (i.e., the quantities in the parenthesis on the right hand side of Equations (5.1) and (5.2)).

\section{Results}

\subsection{Steady Problem}

We consider a steady diffusion problem with $s_{1}=\nu u_{0} \omega^{2} \sin (\omega x)$, so that the exact solution is given by

$$
u=u_{0} \sin (\omega x),
$$

and the exact gradient $p$ is given by the derivative of $u$. The parameters are set as $u_{0}=1.531, \omega=2.423$, $\nu=2.123, L_{r}=1 /(2 \pi), C F L=0.95$. The boundary condition is imposed on $u$ by the exact solution at both ends and $p$ is determined by the scheme as described in Section 3.3. Steady convergence is taken to be achieved when the $L_{1}$ norm of the residual is reduced by 10 orders of magnitude. The initial solution is set by a randomly perturbed exact solution. Computations have been performed for a series of randomly perturbed irregular grids: $32,64,128,256,512,1024$ cells.

The solution and gradient obtained on the coarsest grid are shown in Figure 3. The error and iterative convergence results are plotted in Figure 4. The left figure shows that the scheme is third-order accurate for both the solution $u$ and the gradient $p$, including $p$ on the boundaries. Third-order accuracy is achieved for both the cell-averages and the face-values. The right figure shows the number of pseudo time steps (iterations) required to reduce the residual by 10 orders of magnitude. Clearly, it increases linearly with the grid size (just like advection schemes), not quadratically as typical for conventional diffusion schemes. This $O(1 / h)$ acceleration in iterative convergence is one of the advantages of the hyperbolic method.

\subsection{Unsteady Problem}

We consider an unsteady diffusion problem, $\partial_{t} u=\nu \partial_{x x} u$, with the following boundary conditions:

$$
\begin{aligned}
& u(0)=0, \\
& u(1)=U \cos (\omega t) .
\end{aligned}
$$

The exact solution is given by

$$
\begin{aligned}
u(x, t) & =Q[\sinh (k x) \cos (k x) \sinh (k) \cos (k)+\cosh (k x) \sin (k x) \cosh (k) \sin (k)] \cos (\omega t) \\
& +Q[\sinh (k x) \cos (k x) \cosh (k) \sin (k)-\cosh (k x) \sin (k x) \sinh (k) \cos (k)] \sin (\omega t),
\end{aligned}
$$



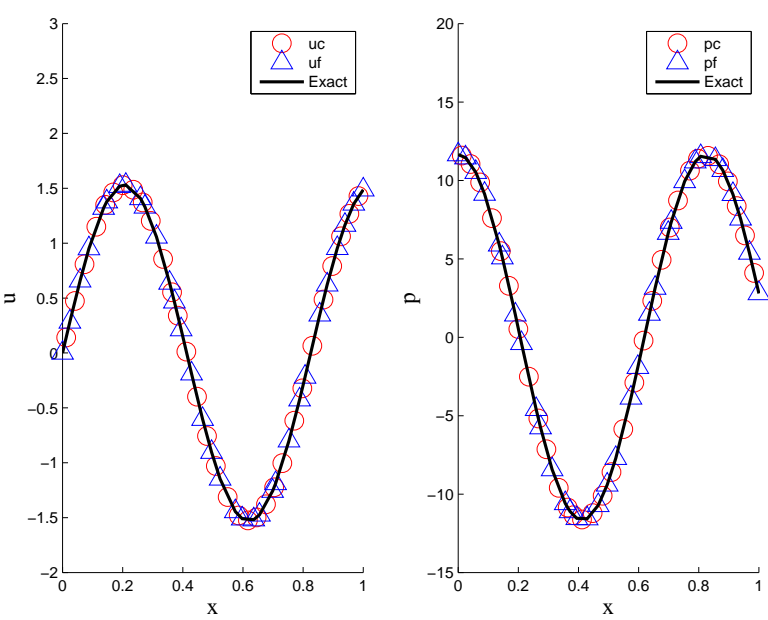

Figure 3. Steady solution on the 32-cell grid. $(u c, p c)$ are the cell-averaged values, and $(u f, p f)$ are the point values at the face. The grid is irregular with the minimum mesh spacing 0.001 .
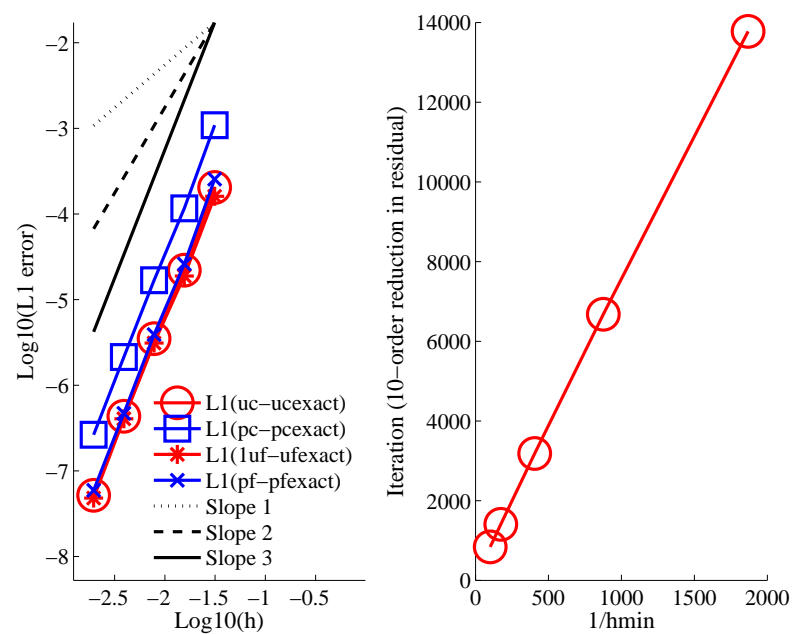

Figure 4. $L_{1}$ Error and iterative convergence results for a steady problem. $(u c, p c)$ are the cell-averaged values, and $(u f, p f)$ are the point values at the face. $h$ is the average mesh spacing, and hmin is the minimum mesh spacing.

where

$$
k=\sqrt{\frac{\omega}{2 \nu}}, \quad Q=\frac{U}{\cosh ^{2}(k)-\cos ^{2}(k)},
$$

and $U$ and $\omega$ are arbitrary constants. See Ref.14 for the derivation. For numerical results, the parameters have been set as follows:

$$
\nu=2.123, \quad U=2.0, \quad \omega=2 \pi, \quad .
$$

The boundary condition is imposed on $u$ by Equation (6.3), and $p$ is determined by the scheme as described before. The CFL number for the sub-iteration is set to be 0.96 . The sub-iteration is taken to be converged when the $L_{1}$ norm of the unsteady residual is reduced by two orders of magnitude. At every physical time step, the initial solution is set as the solution at the previous physical time step. To start up the computation, we use BDF1 over the first step, BDF2 in the next step, and BDF3 thereafter. Ideally, it would be best to perform the first two steps with a small enough time step not to introduce large errors, but numerical results show that the low-order errors in the first two steps do not greatly impact the accuracy of the solution at a later time.

First, we tested the time accuracy for a given grid of 64 cells with randomly distributed nodes by refining the time step: $0.75 / 2^{m}$, where $m=0,1,2,3$. The final time is 6.0 , i.e., 6 periods in the unsteady boundary condition. Results are shown for BDF2 and BDF3 in Figures 5 and 6 , respectively. These figures show that the formal time accuracy has been confirmed. The accuracy deteriorates for BDF3 in the finest grid apparently because the spatial error begins to dominate. The number of sub-iterations increases with the physical time step as expected. It appears to reach the limit of steady convergence.

Second, we performed a time-accurate computation with $\Delta t=0.025$ and the grid of 24 cells. The grid is, again, irregular with the nodes randomly distributed. The final time is 10.0, i.e., 10 periods, which is reached at 400 time steps. The cell-averaged solution and gradient at $t=8.0,8.25,8.5,8.75$ are plotted in Figures 7,8 , 9,10 , respectively. These results show that the active-flux hyperbolic diffusion scheme enables highly accurate unsteady simulations on a rather coarse grid even for irregular grids. For this case, the average number of sub-iterations was $60\left(h_{\min }=0.0192\right)$. The number of sub-iterations depends on the minimum cell volume, which is randomly small in these tests. For uniform grids, the sub-iteration converges much faster, e.g., 36 on average for the second test.

\section{Concluding Remarks}

In this paper, we constructed active-flux schemes for diffusion. The diffusion term has been discretized in the form of a first-order hyperbolic system, which is equivalent to the diffusion equation in the steady 

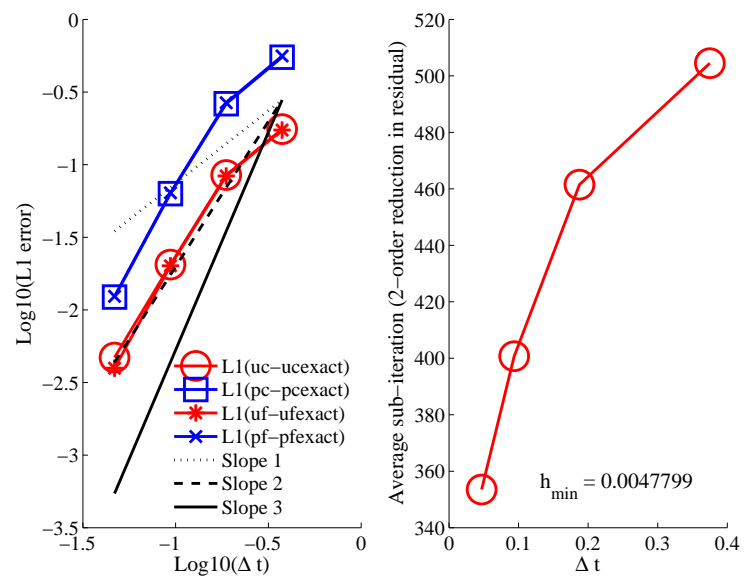

Figure 5. Numerical results for BDF2. Time accuracy on the left and the number of sub-iterations on the right.
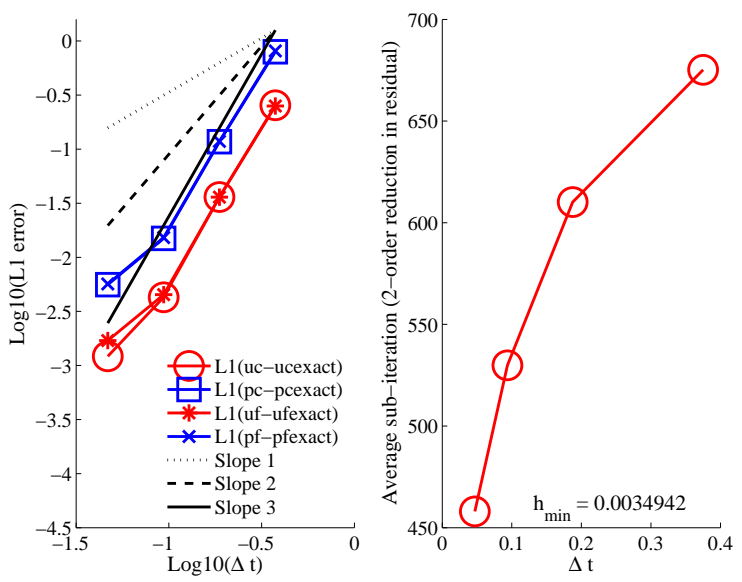

Figure 6. Numerical results for BDF3. Time accuracy on the left and the number of sub-iterations on the right.

state. Construction of active flux schemes for diffusion is then straightforward since the scheme developed for hyperbolic systems is directly applicable. A steady-state solver is constructed based on explicit pseudo-time stepping. Numerical results show that third-order accuracy is obtained for both the solution and the gradient in the steady state. Unsteady schemes are then constructed by the backward-difference formulas, where the steady solver is used to reduce the unsteady residual by two orders of magnitude over each physical time step. Numerical results demonstrate that formal third-order accuracy can be achieved and highly accurate unsteady solutions can be obtained on irregular grids.

Future work will focus on the extension to the advection-diffusion equation and the development of implicit steady solvers. A straightforward extension is possible by the unified hyperbolic formulation for the advectiondiffusion equation as in Ref.6. However, such a unified formulation is not yet possible for the compressible NavierStokes equations. To enable the extension to the compressible Navier-Stokes equations, a separate treatment of the advective and diffusive terms will be required as proposed in Ref.7. Also, there is an alternative approach to the source term discretization based on the divergence formulation of source terms proposed in Ref.15, which may turn out to further simplify the algorithm. In this study, an explicit sub-iteration has been employed, but it may be inefficient for advection-diffusion problems on stretched grids. An implicit solver is expected to improve the efficiency, and should be developed.
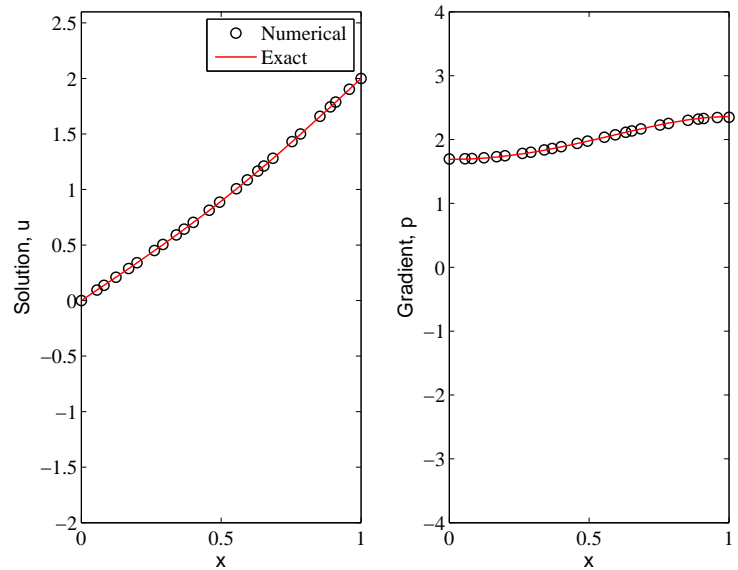

Figure 7. Unsteady solution at $t=8.0 . \Delta t=0.05$ and 24 cells.
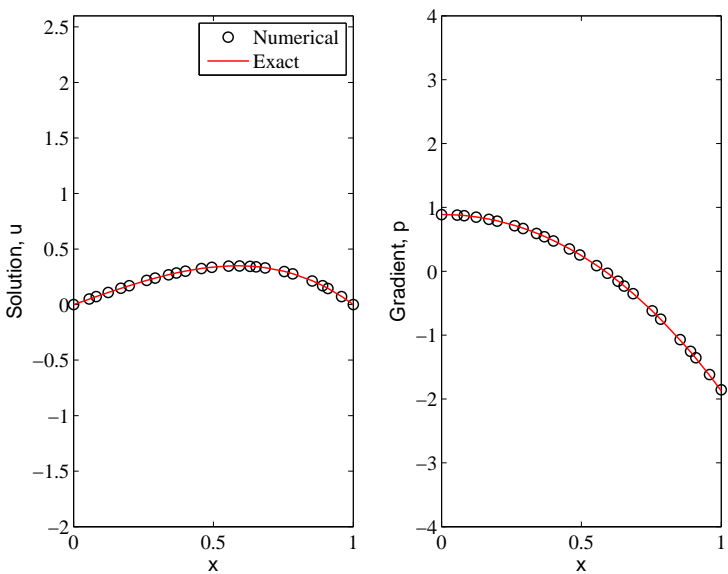

Figure 8. Unsteady solution at $t=8.25 . \Delta t=0.05$ and 24 cells. 

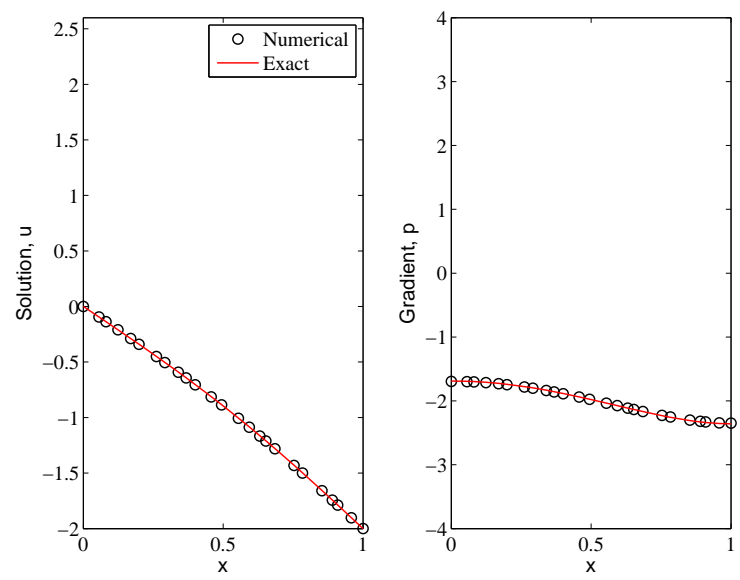

Figure 9. Unsteady solution at $t=8.5 . \Delta t=0.05$ and 24 cells.
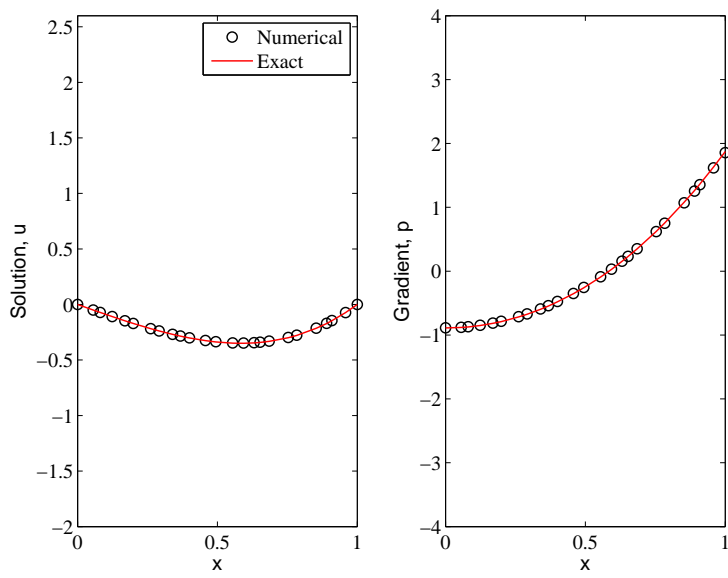

Figure 10. Unsteady solution at $t=8.75 . \Delta t=0.05$ and 24 cells.

\section{Acknowledgments}

This work has been funded by the NASA NRA/ROA-2011 Contract NNX12AJ70A.

\section{References}

${ }^{1}$ Timothy A. Eymann and Philip L. Roe. Active flux schemes for systems. In Proc. of 20th AIAA Computational Fluid Dynamics Conference, AIAA Paper 2011-3840, Hawaii, 2011.

${ }^{2}$ Timothy A. Eymann and Philip L. Roe. Multidimensional active flux schemes. In Proc. of 21st AIAA Computational Fluid Dynamics Conference, AIAA Paper 2013-2940, San Diego, California, June 2013.

${ }^{3}$ B. van Leer and S. Nomura. Discontinuous Galerkin for diffusion. In Proc. of 17th AIAA Computational Fluid Dynamics Conference, AIAA Paper 2005-5108, Toronto, 2005.

${ }^{4}$ M. Sun and P. L. Roe. Viscous active flux method. Unpublished, June 2013.

${ }^{5}$ H. Nishikawa. A first-order system approach for diffusion equation. I: Second order residual distribution schemes. J. Comput. Phys., 227:315-352, 2007.

${ }^{6} \mathrm{H}$. Nishikawa. A first-order system approach for diffusion equation. II: Unification of advection and diffusion. J. Comput. Phys., 229:3989-4016, 2010.

${ }^{7}$ H. Nishikawa. New-generation hyperbolic Navier-Stokes schemes: $O(1 / h)$ speed-up and accurate viscous/heat fluxes. In Proc. of 20th AIAA Computational Fluid Dynamics Conference, AIAA Paper 2011-3043, Honolulu, Hawaii, 2011.

${ }^{8}$ A. Mazaheri and H. Nishikawa. First-order hyperbolic system method for time-dependent advection-diffusion problems. NASA-TM-2014-218175, March 2014.

$\checkmark{ }^{9}$ A. Mazaheri and H. Nishikawa. Very efficient high-order hyperbolic schemes for time-dependent advection-diffusion problems: Third-, Fourth, and Sixth-Order. Comput. Fluids, 2014. in review.

${ }^{10}$ H. Nishikawa. First-, second-, and third-order finite-volume schemes for diffusion. J. Comput. Phys., 256:791-805, 2014.

${ }^{11} \mathrm{H}$. Nishikawa. First, second, and third order finite-volume schemes for advection-diffusion. J. Comput. Phys., 273:287-309, 2014.

${ }^{12} \mathrm{H}$. Nishikawa. First, second, and third order finite-volume schemes for Navier-Stokes equations. In Proc. of 44th AIAA Fluid Dynamics Conference, AIAA Paper 2014-2091, Atlanta, 2014.

${ }^{13}$ N. D. Melson, M. D. Sanetrik, and E. L. Atkins. Time accurate Navier-Stokes calculations with multigrid acceleration. In Proc. of 6th Copper Mountain Conference on Multigrid Methods, (NASA CP 3224), Copper Mountain, 1993.

${ }^{14}$ Katate Masatsuka. I do like CFD, VOL.1, Second Edition. http://www.cfdbooks.com, 2013.

${ }^{15}$ H. Nishikawa. Divergence formulation of source term. J. Comput. Phys., 231:6393-6400, 2012. 